\title{
Synchronous Lung Adenocarcinoma and Papillary Thyroid Carcinoma The Metastases at the Centre of the Discussion
}

\author{
Joana Ferra, Cátia Guimarães, Cristina Matos, Fernando Nogueira \\ Pneumology Department, Hospital Egas Moniz, Centro Hospitalar Lisboa Ocidental E.P.E., Portugal
}

Received: 12/01/2021

Accepted: $17 / 01 / 2021$

Published: 05/02/2021

\begin{abstract}
How to cite this article: Ferra J, Guimarães C, Matos C, Nogueira F. Synchronous lung adenocarcinoma and papillary thyroid carcinoma - the metastases at the centre of the discussion. EJCRIM 2021;8: doi:10.12890/2021_002289.
\end{abstract}

Conflicts of Interests: The Authors declare that there are no competing interests.

This article is licensed under a Commons Attribution Non-Commercial 4.0 License

\section{ABSTRACT}

Synchronous tumours are defined as two or more independent primary neoplasms of different origins diagnosed at the same time in 1 individual. Although rare, its incidence is increasing and the proper diagnosis and staging of each tumour is crucial in defining the patient prognosis and the best therapeutic choice. We present a case of a 56-year-old woman presenting with a lung adenocarcinoma and pulmonary metastases initially diagnosed as stage IV and who was started on a tyrosine kinase inhibitor (erlotinib). In the meantime, she was also diagnosed with papillary thyroid carcinoma and was submitted to complete thyroidectomy. After 6 cycles of erlotinib, thoracic CT showed a decrease in the dimensions of the primary pulmonary tumour, but an increase in the size and number of pulmonary metastases while blood tests showed elevated thyroglobulin. This therefore raised the possibility that the metastases could have originated from the thyroid carcinoma. Anatomo-pathological examination of the lung metastases confirmed this hypothesis. In conclusion, it is important to confirm the origin of metastases in synchronous tumours given this can lead to a re-staging of tumours and a different prognosis, along with other therapeutic options. A multidisciplinary team meeting is crucial to define management and therapeutic approaches for these patients.

\section{LEARNING POINTS}

- Although rare, synchronous primary tumours are a possibility and physicians should be aware of this possibility.

- Papillary thyroid carcinoma may metastasize to the lungs and its differential diagnosis should be made in the presence of lung carcinoma.

- When in doubt, anatomo-pathological examination of the metastases should be performed for proper staging of both tumours and to decide on the best therapeutic option.

\section{KEYWORDS}

Synchronous tumours, lung metastases, papillary thyroid carcinoma, lung adenocarcinoma

\section{CASE DESCRIPTION}

A 56-year-old female patient, non-smoker, with no relevant medical history underwent a chest radiograph that revealed a nodular formation on the right lung field. Thoracic computed tomography (CT) showed a ground-glass nodule on the right lower lobe, measuring $3.4 \times 2$ centimetres and multiple micronodules on the middle lobe, lingula and left lower lobe. Positron emission tomography-CT (PET-CT) showed a hypermetabolic right lower nodule (SUV 5.5) and hypermetabolic lung micronodules compatible with pulmonary metastases. PET-CT also revealed a hypermetabolic thyroid mass and the patient was sent to the Endocrinology Department for further investigation. She underwent CT-guided transthoracic lung biopsy of the ground-glass nodule, which revealed a lepidic adenocarcinoma of the lung. The patient was therefore diagnosed with stage IV lung adenocarcinoma due to pulmonary metastases. 
The tumour had no PD-L1 expression and molecular studies revealed a mutation in epidermal growth factor receptor (EGFR) in exon 21. The patient started tyrosine kinase inhibitor treatment with erlotinib. After 6 cycles of erlotinib, thoracic CT showed a decrease in the dimensions of the primary pulmonary tumour, but an increase in the size and number of pulmonary metastases (Fig. 1).

The patient chose another institution for investigation of the thyroid mass and was then diagnosed with papillary thyroid carcinoma. She underwent complete thyroidectomy and was considered treated. However, during follow-up elevated thyroglobulin was detected (2813cut-off $77 \mathrm{ng} / \mathrm{ml})$.

Given the increase in the size and number of pulmonary metastases (although the primary pulmonary tumour had responded to the tyrosine kinase inhibitor) and elevated thyroglobulin, it was considered that the pulmonary metastases could be due to papillary thyroid carcinoma instead. The patient was submitted to lung surgery and the extemporaneous examination of the pulmonary metastases showed they were related to thyroid carcinoma and not to lung adenocarcinoma. Therefore, the lung adenocarcinoma was in fact at an early stage (stage IB) and she was submitted to right lower lobectomy at the same surgical time. In conclusion, the patient was diagnosed with two synchronous tumours: stage IB lung adenocarcinoma, surgically treated and with no signs of relapse up to this point, and stage IV papillary thyroid carcinoma. For the thyroid carcinoma the patient started lenvatinib (she did not tolerate radioactive iodine) with a radiologic response showing reduction in the size and number of pulmonary metastases (Fig. 2) and maintains follow-up at the Oncology Department.

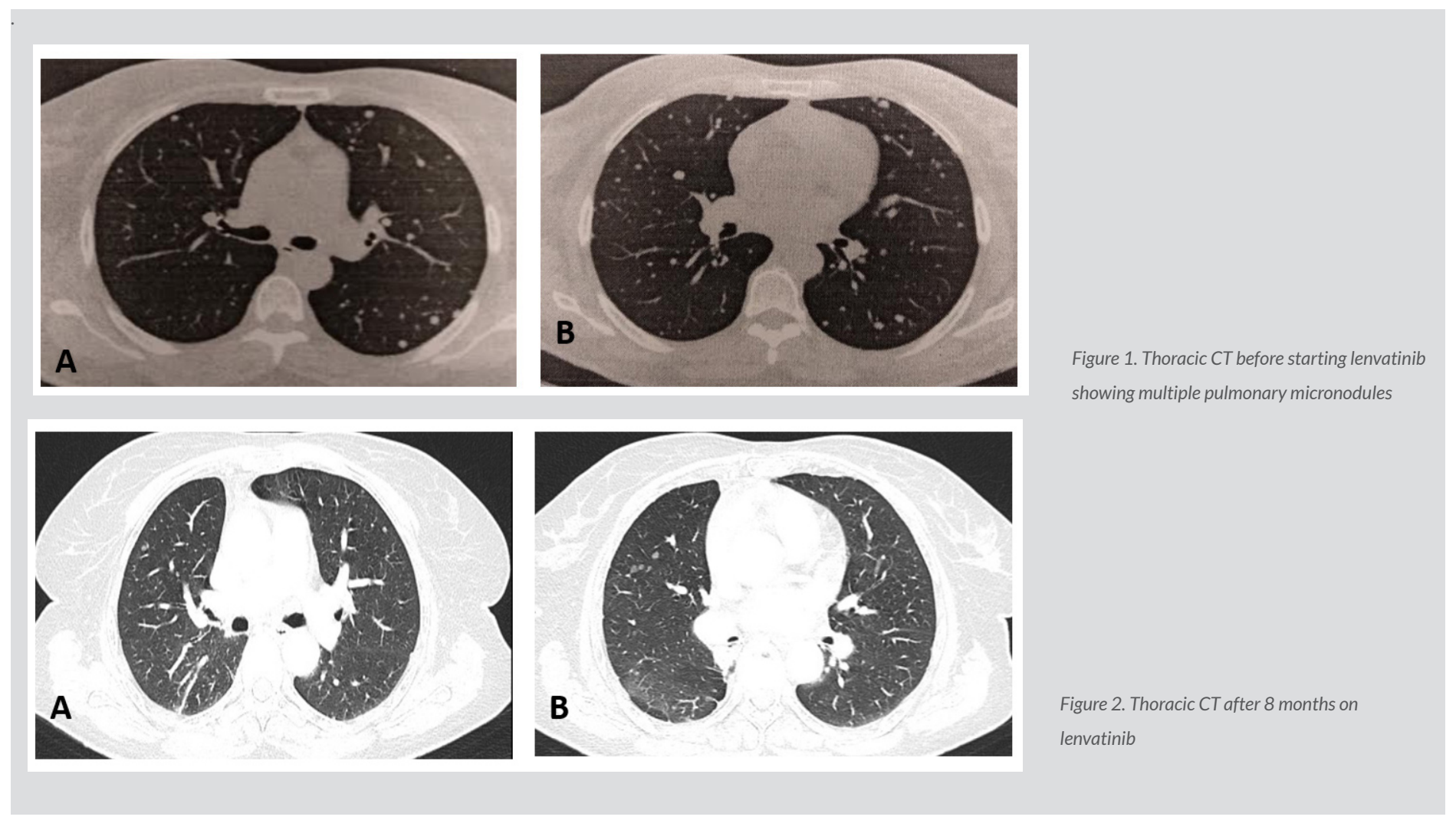

\section{DISCUSSION}

Thyroid carcinomas are a rare but increasingly common entity, accounting for $1 \%$ of all new neoplasms and are more frequent among women $^{[1]}$. The majority of thyroid carcinomas are differentiated thyroid carcinoma (DTC), papillary and follicular carcinoma being the most common types ${ }^{[1]}$. Overall, papillary thyroid carcinoma is relatively indolent, with an overall survival rate of $98 \%{ }^{[1]}$. In DTC, distant metastases are uncommon, with the main sites being the lymph nodes, bone and lungs ${ }^{[2]}$. In patients with metastatic disease, factors related to poor prognosis include older age at diagnosis, histology of the tumour (papillary has a better prognosis than follicular), the site and number of the metastasis/es and 131/ nonavidity ${ }^{[2]}$. In pulmonary metastatic disease, long-term survival is higher in patients with micronodular pulmonary metastases ${ }^{[1]}$. Ten-year survival of patients with papillary thyroid carcinoma and lung metastases varies between $70-93 \%$ according to multiple studies ${ }^{[2]}$.

Conversely, lung carcinoma is 1 of the leading causes of cancer-related mortality worldwide. Non-small cell lung cancer (adenocarcinoma being the most frequently occurring histologic type) accounts for $85 \%$ of all lung cancer cases, and more than half are diagnosed at stage 
IV ${ }^{[3]}$. Five-year survival in metastatic lung cancer is approximately 4-6\%, although new therapies such as tyrosine kinase inhibitors and immune checkpoint inhibitors are allowing these patients to live longer ${ }^{[3,4]}$.

Our patient presented with synchronous malignant tumours, but with an early stage lung adenocarcinoma and metastatic papillary thyroid carcinoma, which is not the most usual presentation and was a confusing factor regarding the choice of treatment. Several factors contributed to misdiagnosis of the origin of the pulmonary metastases. First, the patient was diagnosed with lung adenocarcinoma, an aggressive tumour that is often diagnosed in stage IV and micronodular pulmonary metastases are a frequent presentation. Second, the patient chose to investigate the thyroid mass in another institution and was diagnosed with an indolent thyroid carcinoma that rarely metastasizes and was considered cured without need for further therapeutics. Therefore, given the odds, we decided to treat the lung adenocarcinoma as stage IV and started the tyrosine kinase inhibitor according to international recommendations. It was only when we saw an opposite behaviour regarding the pulmonary primary tumour and pulmonary metastases, along with elevated thyroglobulin, that we considered the possibility that the metastases originated from thyroid carcinoma. This possibility was confirmed by anatomo-pathologic examination of the metastases, which led to a re-staging of both tumours. Retrospectively, we consider it would be important to confirm the origin of the metastases given the patient was diagnosed with two synchronous tumours that have a different prognosis and therapeutic options, in order to make the best therapeutic choice regarding both cancers. Moreover, this case highlights the importance of multidisciplinary team meetings between physicians from different Departments (in our case, the Pulmonology Department for follow-up of lung adenocarcinoma and the Oncology Department for follow-up of thyroid carcinoma) to determine the most optimal choice for the patient.

\section{REFERENCES}

1. Sherman S. Thyroid carcinoma. Lancet 2003;361:501-511.

2. Cho SW, Choi HS, Yeom GJ, Lim JA, Moon JH, Park DJ, et al. Long-term prognosis of differentiated thyroid cancer with lung metastasis in Korea and its prognostic factors. Thyroid 2014;24:277-286.

3. Molina JR, Yang P, Cassivi SD, Schild SE, Adjei AA. Non-small cell lung cancer: epidemiology, risk factors, treatment, and survivorship. Mayo Clin Proc 2008;83:584-594.

4. Cetin K, Ettinger DS, Hei YJ, O'Malley CD. Survival by histologic subtype in stage IV nonsmall cell lung cancer based on data from the surveillance, epidemiology and end results program. Clin Epidemiol 2011;3:139-148. 